\title{
Double blind peer review
}

Public Domain

\section{Source}

Open Research Glossary

When the reviewers don't know who the authors are, and vice versa. 